H. Matsumura

Nagoya Math. J.

Vol. 68 (1977), 123-130

\title{
QUASI-COEFFICIENT RINGS OF A LOCAL RING
}

\author{
HIDEYUKI MATSUMURA
}

In this note we will make a few observations on the structure of fields and local rings. The main point is to show that a weaker version of Cohen structure theorem for complete local rings holds for any (not necessarily complete) local ring. The consideration of non-complete case makes the meaning of Cohen's theorem itself clearer. Moreover, quasicoefficient fields (or rings) are handy when we consider derivations of a local ring.

1. All rings considered here are commutative rings with unit element. By a local ring $(A, \mathfrak{m})$ we mean a (not necessarily noetherian) ring $A$ with unique maximal ideal $\mathfrak{m}$. The completion of $(A, \mathfrak{m})$ is $\lim _{\leftarrow} A / \mathfrak{m}^{n}$ and is denoted by $A^{*}$. We say that $A$ is separated if $\cap_{n} \mathfrak{m}^{n}$ $=(0)$, and that $A$ is complete if $A=A^{*}$.

Let $(A, \mathfrak{m})$ and $(B, \mathfrak{n})$ be noetherian local rings and $\phi: A \rightarrow B$ be a local homomorphism. Then $B$ is said to be formally smooth (resp. formally unramified, resp. formally etale) over $A$ if, for every commutative diagram

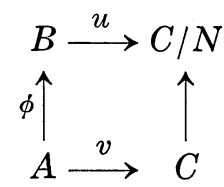

where $C$ is a ring, $N$ is an ideal of $C$ with $N^{2}=(0)$ and $u\left(\mathfrak{m}^{r}\right)=(0)$ for sufficiently large $r$, there exists at least one (resp. at most one, resp. exactly one) homomorphism $B \rightarrow C$ which makes the diagram

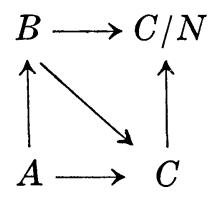

Received February 16, 1977. 
commutative (cf. $[3, \S 19])$.

If $B$ is formally unramified over $A$, then $\operatorname{Der}_{A}(B, M)=0$ for any $B$-module $M$ such that $\bigcap_{\nu} \mathfrak{n}^{\nu} M=0$. In particular, if we put $k=A / \mathfrak{m}$ and $K=B / \mathfrak{n}$, then $\operatorname{Der}_{k}(K)=0$ (or what is the same, $\Omega_{K / k}=0$ ). On the other hand, it is not difficult to show that if $\Omega_{K / k}=0$ and $\mathfrak{n}=\mathfrak{m} B$ then $B$ is formally unramified over $A$.

$A$ necessary and sufficient condition for $B$ to be formally smooth over $A$ is that (1) $B$ is flat over $A$ and (2) $B / \mathfrak{m} B$ is formally smooth over $A / \mathfrak{m}$ [3, (19.7.1)]. If $A$ and $B$ are fields, then to say $B$ is formally smooth over $A$ is tantamount to saying that $B$ is separable over $A$.

Let $K$ be a field and $k$ be a subfield. Then the following conditions are equivalent:

(a) $K$ is formally etale over $k$;

(b) every derivation of $k$ into a $K$-module $M$ can be uniquely extended to a derivation of $K$ into $M$;

(c) $\Omega_{K}=\Omega_{k} \otimes_{k} K$, where $\Omega_{k}$ denotes the module of differentials of $k$ over the prime field;

(d) $K$ is separable over $k$ and $\Omega_{K / k}=0$;

(e) $\operatorname{char}(k)=0$ and $K$ is algebraic over $k$; or $\operatorname{char}(k)=p>0$ and a $p$-basis of $k$ (over the prime field) is also a $p$-basis of $K$;

In the case of characteristic $p$, the above are also equivalent to

(f) $K=k \otimes_{k p} K^{p}$.

THEOREM 1. Let $k$ be a field of characteristic $p$, and $K$ be a separable extension of $k$; let $B=\left\{b_{i}\right\}_{i \in I}$ be a p-independent subset of $K$ over $k$. Then $B$ is algebraically independent over $k$.

Proof. Assume the contrary and suppose $b_{1}, \cdots, b_{n} \in B$ are algebraically dependent over $k$. Take an algebraic relation

$$
f\left(b_{1}, \cdots b_{n}\right)=0, \quad f \in k\left[X_{1}, \cdots, X_{n}\right]
$$

of lowest possible degree. Put $\operatorname{deg} f=d$. We can write

$$
f\left(X_{1}, \cdots, X_{n}\right)=\sum_{0 \leqslant \nu_{1}, \cdots \nu_{n}<p} g_{\nu_{1}, \ldots, \nu_{n}}\left(X_{1}^{p}, \cdots, X_{n}^{p}\right) X_{1}^{\nu_{1}} \cdots X_{n}^{\nu_{n}},
$$

where $g_{\nu_{1}, \ldots, \nu_{n}}$ are polynomials with coefficients in $k$. Since $b_{1}, \cdots, b_{n}$ are $p$-independent over $k$, we must have

$$
g_{\nu_{1}, \cdots, \nu_{n}}\left(b_{1}^{p}, \cdots, b_{n}^{p}\right)=0
$$


for all $\nu_{1}, \cdots, \nu_{n}$. By the choice of $f$ this is possible only if

$$
f\left(X_{1}, \cdots, X_{n}\right)=g_{0, \cdots, 0}\left(X_{1}^{p}, \cdots, X_{n}^{p}\right) .
$$

But then we would have

$$
f\left(X_{1}, \cdots, X_{n}\right)=\phi\left(X_{1}, \cdots, X_{n}\right)^{p} \quad \text { with } \phi \in k^{p-1}\left[X_{1}, \cdots, X_{n}\right] .
$$

Hence $\phi\left(b_{1}, \cdots, b_{n}\right)=0$. By MacLane's criterion of separability, however, $K$ and $k^{p^{-1}}$ are linearly disjoint over $k$; since the monomials of degree $<d$ in $b_{1}, \cdots, b_{n}$ are linearly independent over $k$, they are also linearly independent over $k^{p^{-1}}$. Therefore such a relation as $\phi\left(b_{1}, \cdots, b_{n}\right)$ $=0$ cannot exist, and we get a contradiction.

Remark 1. A $p$-basis of a separable extension $K / k$ need not be a transcendence basis. For example, if $k$ is a perfect field and $x$ is an indeterminate over $k$, then the field $k\left(x, x^{p^{-1}}, x^{p^{-2}}, \cdots\right)$ is perfect, so that the empty set is a $p$-basis of this extension.

Remark 2. Recall that a differential basis $\left\{b_{i}\right\}_{i \in I}$ of a field extension $K / k$ is a subset of $K$ such that $\left\{d b_{i}\right\}_{i \in I}$ is a linear basis of $\Omega_{K / k}$ over $K$. The notion of differential basis coincides with that of transcendence basis if $\operatorname{char}(k)=0$, and with that of $p$-basis if $\operatorname{char}(k)=p$.

THEOREM 2. Let $K / k$ be a separable extension of fields. Then there is a subextension $K^{\prime}$ such that $K^{\prime} / k$ is purely transcendental and $K / K^{\prime}$ is formally etale.

Proof. It suffices to take a differential basis $B$ of $K / k$ and put $K^{\prime}$ $=k(B)$.

2. Definition. Let $(A, \mathfrak{m})$ be a local ring containing a field. A subfield $k$ of $A$ is called a quasi-coefficient field (q.c.f.) of $A$ if the residue field $A / \mathfrak{m}$ is formally etale over $k$.

THEOREM 3. (i) Let $k$ be a q.c.f. of a local ring $(A, \mathfrak{m})$. Then there exists a unique coefficient field $k^{\prime}$ of the completion $A^{*}$ of $A$ such that $k \subset k^{\prime}$.

(ii) If a local ring $(A, \mathfrak{m})$ includes a field $k_{0}$ and if $A / \mathfrak{m}$ is separable over $k_{0}$, then $A$ has a q.c.f. $k$ which includes $k_{0}$.

Proof. (i) This is clear from the definitions and from the following diagram. 


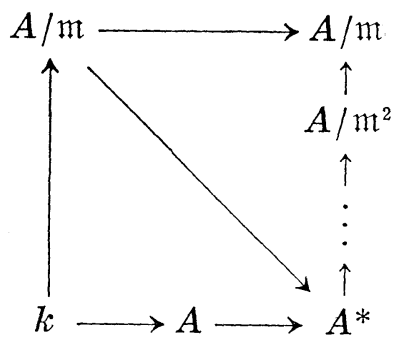

(ii) Let $B$ be a differential basis of $A / \mathfrak{m}$ over $k_{0}$, and choose a preimage $x_{\lambda}$ for each element $b_{\lambda}$ of $B$. If $f\left(X_{1}, \cdots, X_{n}\right)$ is a non-zero polynomial with coefficients in $k_{0}$ and if $b_{1}, \cdots, b_{n}$ are mutually distinct elements of $B$, then $f\left(b_{1}, \cdots, b_{n}\right) \neq 0$ by Theorem 1 , hence $f\left(x_{1}, \cdots, x_{n}\right)$ is invertible in $A$. Therefore $A$ includes the quotient field $k$ of $k_{0}\left[\left\{x_{\lambda}\right\}\right]$, and $k$ is obviously a q.c.f. of $A$.

Remark 3. In the notation of (i), every derivation $D$ of $A$ (into itself) over $k$ is uniquely extended to a derivation of $A^{*}$ over $k^{\prime}$. Therefore we can identify $\operatorname{Der}_{k}(A)$ with an $A$-submodule of $\operatorname{Der}_{k^{\prime}}\left(A^{*}\right)$.

THEOREM 4. Let $(A, \mathfrak{m})$ and $(B, \mathfrak{n})$ be local rings such that $A \subset B$, $\mathfrak{m}=A \cap \mathfrak{n}$. Suppose that $A$ includes a field.

(i) If $B / \mathfrak{n}$ is separable over $A / \mathfrak{m}$, then every q.c.f. of $A$ can be extended to a q.c.f. of $B$.

(ii) If $A$ is of characteristic $p$ and $B^{p} \subset A$, then there exists a q.c.f. of $A$ which can be extended to a q.c.f. of $B$.

Proof. (i) Immediate from (ii) of Theorem 3.

(ii) Put $K=A / \mathfrak{m}$ and $L=B / \mathfrak{n}$. Then $L^{p} \subset K \subset L$. Let $B=\left\{\beta_{i}\right\}_{i \in I}$ be a $p$-basis of $L / K$ and $C=\left\{\gamma_{j}\right\}_{j \in J}$ be a $p$-basis of $K / L^{p}$. Then it is easy to see that $\left\{\gamma_{j}\right\} \cup\left\{\beta_{i}^{p}\right\}$ is a $p$-basis of $K$ and $\left\{\beta_{i}\right\} \cup\left\{\gamma_{j}\right\}$ is a $p$-basis of $L$. Therefore, if $\left\{y_{i}\right\}$ (resp. $\left\{z_{j}\right\}$ ) is a set of representatives of $\left\{\beta_{i}\right\}$ in $L$ (resp. of $\left\{\gamma_{j}\right\}$ in $\left.K\right)$, then $\boldsymbol{F}_{p}\left(\left\{y_{i},\left\{z_{j}\right\}\right)\right.$ is a q.c.f. of $L$ and $\boldsymbol{F}_{p}\left(\left\{z_{j}\right\},\left\{y_{i}^{p}\right\}\right)$ is a q.c.f. of $K$. (cf. Nagata [6]).

THEOREM 5. Let $A$ be a noetherian local integral domain of characteristic $p$, and let $K$ be the quotient field of $A$. Suppose $A$ is pseudogeometric (i.e. Nagata ring in the terminology of [4]). Let $A^{*}$ be the completion of $A, \mathfrak{p}$ be a minimal prime ideal of $A^{*}$ and $L$ be the quotient field of $A^{*} / p$. Let $k$ be a q.c.f. of $A$ and $k^{\prime}$ be the coefficient field of $A^{*}$ including $k$. Then $K$ is separable over $k$ if and only if $L$ is 
separable over $k^{\prime}$.

Proof. Since $A$ is pseudo-geometric, $L$ is separable over $K$ [4, (31. F)]. Suppose $K$ is separable over $k$. Then $L$ is separable over $k$. Let $d$ be a derivation of $k^{\prime}$ into $L$, and let $d_{0}$ denote the restriction of $d$ to $k$. Then $d_{0}$ can be extended to a derivation $D: L \rightarrow L$. The restriction $D \mid k^{\prime}$ must coincide with $d$, since $k^{\prime}$ is formally etale over $k$. Therefore $D$ is an extension of $d$ to $L$. This proves that $L$ is separable over $k^{\prime}$. The converse is easy, since a subextension of a separable extension is separable.

Remark 4. Chevalley [8] gave the following definitions. Let $\mathfrak{o}$ be a noetherian complete local ring which includes a field $k$, and $u_{1}, u_{2}, \ldots$ be a sequence of elements of $\mathfrak{o}$ which converges to 0 in $\mathfrak{D}$. If the conditions $\sum a_{i} u_{i}=0, a_{i} \in k$, imply $a_{i}=0$ for all $i$, then the elements $u_{i}$ are said to be strongly linearly independent over $k$. The elements of a finite sequence are said to be strongly linearly independent over $k$ when they are linearly independent. When $\operatorname{char}(\mathfrak{o})=p$, we will say that $\mathfrak{o}$ is strongly separable ${ }^{1)}$ over $k$ if, for every finite or infinite sequence $\left(u_{i}\right)$ of elements of $\mathfrak{D}$ which are strongly linearly independent over $k$, the elements $u_{i}^{p}$ are strongly linearly independent over $k$. Suppose $\mathfrak{o}$ is an integral domain and let $L$ denote its quotient field. Then clearly

$\mathcal{D}$ is strongly separable over $k \Rightarrow L$ is separable over $k$. It is easy to see that the converse is also true if $\left[k: k^{p}\right]<\infty$, but in general the two conditions are not equivalent. Under the assumption that the residue field of $\mathfrak{o}$ is a finite algebraic extension of $k$, a noetherian complete local domain $\mathfrak{b}$ is strongly separable over $k$ if and only if there exists a system of parameters $x_{1}, \cdots, x_{n}$ of o such that $L$ is separable over the quotient field $k\left(\left(x_{1}, \cdots, x_{n}\right)\right)$ of $k\left[\left[x_{1}, \cdots, x_{n}\right]\right]$ (Nagata [7]). It is desirable to study quasi-coefficient fields further in the direction of Theorem 5 taking these definitions and facts into consideration.

3. In the unequal characteristic case we must define quasi-coefficient ring. Let us recall that, when $(A, \mathfrak{m})$ is a complete local ring with $\operatorname{char}(A / \mathfrak{m})=p>0$, a subring $I$ of $A$ is called a coefficient ring of $A$ if (i) $I$ is a noetherian complete local ring with maximal ideal $p I$ (whence $p I=\mathfrak{m} \cap I$ ) and (ii) $A$ and $I$ have the same residue field, i.e. $A=I+\mathfrak{m}$.

1) In Chevalley's terminology $\mathfrak{v}$ is said to be separably generated over $k$. 
DEFINITION. Let $(A, \mathfrak{m})$ be a (not necessarily complete) local ring with $\operatorname{char}(A / \mathfrak{m})=p>0$. A subring $I$ of $A$ is called a quasi-coefficient ring of $A$ if

( $\left.i^{\prime}\right) \quad I$ is a noetherian local ring with maximal ideal $p I$, and

(ii') the residue field $A / \mathfrak{m}$ of $A$ is formally etale over $I / p I$.

In both cases, all ideals of $I$ have the form $p^{m} I(m \geqslant 0)$. Therefore, if $\operatorname{char}(A)=0$ (i.e. the unique homomorphism $Z \rightarrow A$ is injective) then $p^{m} I \neq 0$ for all $m \geqslant 0$ and $I$ is a discrete valuation ring. If char $(A)$ $=p^{n}, n>0$, then we have $p^{n-1} I \neq 0, p^{n} I=0$ and $I$ is artinian.

Remark 5. In the case $\operatorname{char}(A)=p^{n}$, there exists a complete discrete valuation ring $W$ with maximal ideal $p W$ such that $I \cong W / p^{n} W$, and such $W$ is uniquely determined. In fact, for each field $k$ of characteristic $p$ there exists a complete discrete valuation ring $W$ of characteristic zero such that $W / p W \cong k$, and such $W$ is necessarily flat over $Z_{p Z}$, hence is unique up to isomorphism [3, (19.7.2)]. Moreover, $W$ is formally smooth over $Z_{p Z}$ by [3, (19.7.1)], hence for any complete local ring $\left(B, \mathfrak{m}_{B}\right)$ with residue field $k$ there exists at least one homomorphism $W \rightarrow B$ which lifts the isomorphism $W / p W \cong B / \mathfrak{m}_{B}$. The ring $I$ considered above with maximal ideal $p I$ such that $p^{n-1} I \neq 0, p^{n} I=0$, is artinian, hence complete, and if we take $I$ for $B$ then the homomorphism $W \rightarrow I$ is surjective with kernel $p^{n} W$.

THEOREM 6. Let $(A, \mathfrak{m})$ be a noetherian local ring and $A^{*}$ be its completion. Let $I$ be a quasi-coefficient ring of $A$. Then there exists a unique coefficient ring $J$ of $A^{*}$ including $I$, and $J$ is formally unramified over I. If $A$ is flat over $I$, then $A^{*}$ is flat over $J$ and $J$ is formally etale over $I$.

Proof. Since $A$ is separated, we may view $A$ and $I$ as subrings of $A^{*}$. By [3, (19.7.2)] there exists a complete noetherian local ring $J^{\prime}$ and a flat local homomorphism $I \rightarrow J^{\prime}$ such that $J^{\prime} / p J^{\prime} \cong A / \mathfrak{m}$ over $I / p I$. Since $\operatorname{rad}\left(J^{\prime}\right)=p J^{\prime}=\operatorname{rad}(I) J^{\prime}$ and since $J^{\prime} / p J^{\prime}$ is formally etale over $I / p I$, it is easy to see that $J^{\prime}$ is formally etale over $I$. Therefore there is a unique homomorphism $\phi: J^{\prime} \rightarrow A^{*}$ which makes the following diagram commutative : 


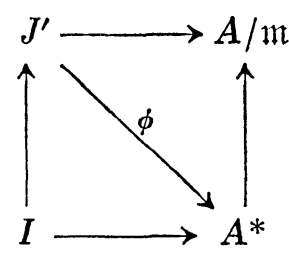

Put $J=\phi\left(J^{\prime}\right)$. Then $J$ is a coefficient ring of $A^{*}$. Since $J^{\prime}$ is formally unramified over $I$, so is $J$. If $A$ is flat over $I$ then $A^{*}$ is also flat over $I$, hence we have

$$
p J^{\prime} \otimes_{J^{\prime}} A^{*}=\left(p I \otimes_{I} J^{\prime}\right) \otimes_{J^{\prime}} A^{*}=p I \otimes_{I} A^{*}=p A^{*} .
$$

Therefore (by [1, Ch. 3, 55 , no. 2, Theorem 1 (iii)], [4, (20.C)]) the map $\phi$ makes $A^{*}$ a flat $J^{\prime}$-module, and consequently $\phi$ is injective (since it is local). Thus $J^{\prime} \cong J$.

It remains to prove the uniqueness of $J$. If $J^{\prime \prime}$ is a coefficient ring of $A^{*}$ including $I$, then we can use the same argument to prove the existence of a homomorphism $\psi: J^{\prime} \rightarrow J^{\prime \prime}$ such that

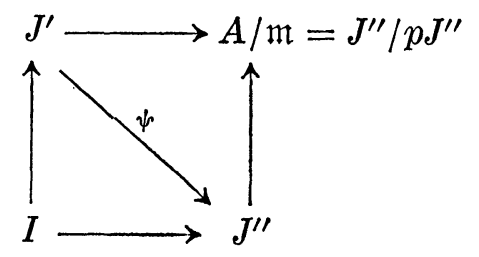

commutes. Let $i: J^{\prime \prime} \rightarrow A^{*}$ denote the inclusion map. Then $\phi=i \circ \psi$ by the uniqueness of $\phi$, hence $J^{\prime \prime}=J$. QED.

Corollary. Let $(A, \mathfrak{m})$ and $I$ be as in the theorem, and let $\left\{y_{\lambda}\right\}$ be a system of generators of $\mathrm{m}$. If $D \in \operatorname{Der}_{I}(A)$ and $D\left(y_{2}\right)=0$ for all $\lambda$, then $D=0$.

Proof. Extend $D$ to $A^{*}$ by continuity. Then $D=0$ on $J$, hence on $A^{*}$.

Quasi-coefficient rings exist in any local ring of unequal characteristic. In fact, our next theorem gives a little stronger existence statement.

THEOREM 7. Let $(A, \mathfrak{m})$ be a local ring, and $(C, \mathfrak{p})$ be a noetherian local ring such that $C \subset A, \mathfrak{p}=\mathfrak{m} \cap C$. Suppose $A / \mathfrak{m}$ is separable over $C / \mathfrak{p}$. Then there is a noetherian local ring $(B, \mathfrak{n})$ such that $C \subset B \subset A$, 
$\mathfrak{n}=\mathfrak{p} B=\mathfrak{m} \cap C$ and such that $A / \mathrm{m}$ is formally etale over $B / \mathfrak{n}$. If $A$ is flat over $C$, then $A$ is also flat over $B$.

Proof. Let $\left\{\bar{x}_{i}\right\}_{i \in I}$ be a differential basis of $A / \mathfrak{m}$ over $C / \mathfrak{p}$, and let $x_{i} \in A$ be a pre-image of $\bar{x}_{i}$ for each $i \in I$. Let $\left\{X_{i}\right\}_{i \in I}$ be independent variables and put $R=C\left[\left\{X_{i}\right\}\right], B^{\prime}=R_{p R}$. Then $B^{\prime}$ is noetherian. In fact, it is a local ring with finitely generated maximal ideal, and $\cap_{\nu} \mathfrak{p}^{\nu} B^{\prime}=(0)$ because $\left(\cap \mathfrak{p}^{\nu} B^{\prime}\right) \cap R=\cap \mathfrak{p}^{\nu} R=(0)$. Moreover, if $\mathfrak{a}=\left(f_{1}, \cdots, f_{r}\right)$ is a finitely generated ideal of $B^{\prime}$ then $B^{\prime} / \mathfrak{a}$ is also a localization of a polynomial ring over a noetherian local ring, hence $B^{\prime} / a$ is also separated. In other words, every finitely generated ideal of $B^{\prime}$ is closed. It follows that $B^{\prime}$ is noetherian [5, (31.8)].

Consider the $C$-homomorphism $R \rightarrow A$ which maps $X_{i}$ to $x_{i}$. Since $\left\{\bar{x}_{i}\right\}_{i \in I}$ is algebraically independent over $C / \mathfrak{p}$, the homomorphism $R \rightarrow A$ factors as $R \rightarrow B^{\prime} \rightarrow A$. Denote the image of $B^{\prime}$ in $A$ by $B$. Then $B$ is

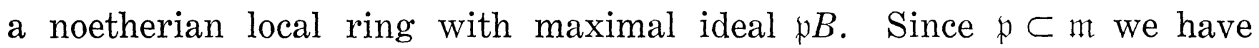
$\mathfrak{p} B=\mathfrak{m} \cap B$. The last assertion of the theorem is proved as in Theorem 6.

If $(A, \mathfrak{m})$ is a local ring with $\operatorname{char}(A / \mathfrak{m})=p>0$, then we can find a local subring $C$ with maximal ideal $p C$ satisfying the condition of Theorem 7. It suffices to take $C=Z_{p Z}$ when $\operatorname{char}(A)=0$, and $C=Z / p^{n}$ when $\operatorname{char}(A)=p^{n}$. Then the local ring $B$ of the theorem is a quasicoefficient ring of $A$.

\section{REFERENCES}

[1] N. Bourbaki, Algèbre Commutative, Ch. 3,4. Hermann, Paris, 1961.

[2] I. S. Cohen, On the structure and ideal theory of complete local rings, Trans. Amer. Math. Soc. 59 (1946), 54-106.

[3] A. Grothendieck and J. Dieudonné, Éléments de Géométrie Algébrique, Ch. IV, Première Partie, Publ. IHES, No. 20, 1964.

[4] H. Matsumura, Commutative Algebra, Benjamin, New York 1970.

[ 5 ] M. Nagata, Local Rings, Interscience, New York 1962.

[6] - Note on coefficient fields of complete local rings. Mem. Coll. Sci., Univ. Kyoto 32 (1959), 91-92.

[7] - Note on complete local integrity domains. Mem. Coll. Sci., Univ. Kyoto 28 (1954), 271-278.

[8] C. Chevalley, Some properties of ideals in rings of power series. Trans. Amer. Math. Soc. 55 (1944), 68-84. 